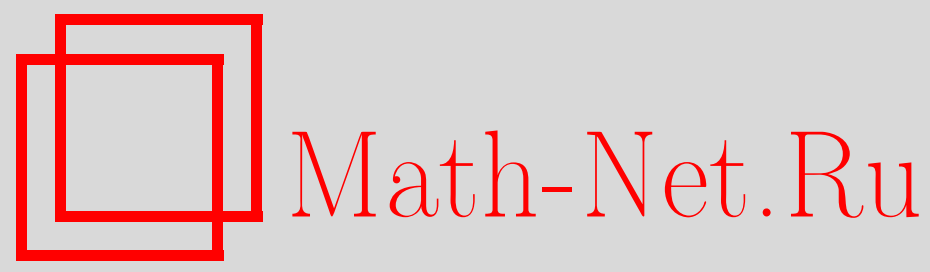

Ю. С. Колесов, Природа буферности, Матем. заметки, 2003, том 74, выпуск 2, 238-241

DOI: https://doi.org/10.4213/mzm260

Использование Общероссийского математического портала Math-Net.Ru подразумевает, что вы прочитали и согласны с пользовательским соглашением http://www .mathnet.ru/rus/agreement

Параметры загрузки:

IP : 54.237 .206 .68

26 апреля 2023 г., 13:44:58

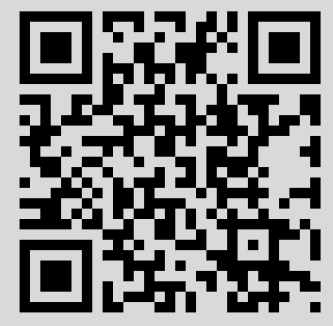




\section{ПРИРОДА БУФЕРНОСТИ}

Ю. С. Колесов

Сформулирована теорема о неустойчивости автомодельных циклов и торов определенного типа в системе, являющейся квазинормальной формой краевой задачи для нелинейного волнового уравнения в квадрате.

Библиограффия: 1 название.

Если нелинейная краевая задача для некоторого волнового уравнения имеет большое число устойчивых пространственно неоднородных циклов, устроенных достаточно однотипно, то принято говорить о явлении буферности. К настоящему времени обнаружено, что при различном выборе краевых условий для волновых уравнений на отрезке данное явление типично. Однако, в плоской области, как показано в [1], где при нулевых граничных условиях рассмотрено часто встречающееся волновое уравнение, явление буферности места не имеет. Поэтому возникает вопрос о природе буферности при других граничньх условиях. По ряду причин наиболее естественньми представляются граничные условия Неймана.

1. Ниже рассматривается бифуркационная проблема для того же уравнения, что и в [1], но при условиях непроницаемости на границе $Г$ некоторой ограниченной плоской области $\Omega$ :

$$
u_{t t}-\varepsilon u_{t}+u^{2} u_{t}+\delta^{2} u=\Delta u,\left.\quad \frac{\partial u}{\partial \nu}\right|_{\Gamma}=0 .
$$

Здесь $\Delta$ - оператор Лапласа, $\varepsilon$ - мальй положительный параметр, $\delta>0$ - постоянная, $\nu$ - направление внешней нормали в произвольной точке $Г$. Фазовым пространством считаем произведение соболевских пространств $\stackrel{\circ}{{ }_{2}^{2}} \times W_{2}^{1}$.

Ограничимся описанием полученных результатов для трех случаев:

$\Omega$ - единичный квадрат, т.е. $0 \leqslant x, y \leqslant 1$;

$\Omega$ - прямоугольник, т.е. $0 \leqslant x \leqslant l_{1}, 0 \leqslant y \leqslant l_{2}, l_{1} \neq l_{2}$;

$\Omega$ - треугольник, задаваемьй неравенствами $x, y \geqslant 0, x+y \leqslant 1$.

Начнем со второго случая, для которого явление буферности имеет место. В этом случае устойчивые циклы одномерны в том смысле, что каждый из них зависит только от одной пространственной переменной.

Наиболее неожиданньм оказалось, что в первом случае буферность связана с наличием большого числа в определенном смысле симметричных пар мод. 
Эти два примера подсказьвают, что, вероятно, явление буферности, если оно в принципе возможно, в отмеченном вьше смысле связано с одномерностью бифурцирующих циклов.

В третьем случае оператор Лапласа не имеет нетривиальных собственньх функций, зависящих от одной пространственной переменной, что объясняет полученньй результат: неустойчивы все пространственно неоднородные циклы и торы, бифурцирующие из нулевого состояния равновесия.

Во втором и третьем пунктах мы кратко опишем доказательство одного результата, связанного с первым случаем.

2. Пусть $\Omega$-квадрат. Тогда если в (1) отбросить нелинейность и положить $\varepsilon=0$, то решения получившейся линейной краевой задачи осциллируют с частотным набором:

$$
\omega_{00}=\delta, \quad \omega_{k n}=\pi \sqrt{\delta^{2}+k^{2}+n^{2}}, \quad k+n>0, \quad k, n=0,1, \ldots
$$

Нормированные собственные функции, по которым естественным образом разлагаются в ряд Фурье решения линейной краевой задачи, соответственно равны

$$
\begin{gathered}
u_{00}=1, \quad u_{k n}=2 \cos k \pi x \cos n \pi y, \quad k, n=1,2, \ldots, \\
u_{k 0}=\sqrt{2} \cos k \pi x, \quad u_{0 n}=\sqrt{2} \cos n \pi y .
\end{gathered}
$$

В соответствии с идеологией метода квазинормальных форм решения краевой задачи (1) представляем в виде

$$
u=\varepsilon^{1 / 2} u_{0}+\varepsilon^{3 / 2} u_{1}+\cdots,
$$

где $u_{0}=\sum\left[v_{k n}(\tau) \exp \left(i \omega_{k n} t\right)+\right.$ к.c. $] u_{k n}(x, y), \tau=\varepsilon t-$ медленное время, к.с. - принятое сокращение для обозначения комплексно сопряженной величины, гладкая функция $u_{1}=u_{1}(\tau, t, x, y)$ гармонична по $t$.

Подставляя (4) в (1) и приравнивая коэффициенты при степенях $\varepsilon^{3 / 2}$, во времени $\tau$ для переменных $v_{k n}$ и $\bar{v}_{k n}$ получаем счетную систему обыкновенных дифференциальных уравнений, структура которых существенно зависит от резонансов третьего порядка частотного набора (2). С учетом специфики задачи в [1] показано, что возможны только тождественные резонансы, к которым, в частности, относится каждое из формальных равенств

$$
\omega_{k n}=2 \omega_{n k}-\omega_{k n}, \quad k+n>0, \quad k, n=0,1, \ldots
$$

Отметим, что для прямоугольной области они невозможны.

Из (3), (5) следует, что в квадрате квазинормальная форма краевой задачи (1) устроена следующим образом:

$$
\begin{aligned}
2 \frac{d v_{k n}}{d \tau}= & v_{k n}-\frac{9}{4} v_{k n}\left|v_{k n}\right|^{2}-\bar{v}_{k n} v_{n k}^{2}-2 v_{k n} \sum_{p \neq k, s \neq n}\left|v_{p s}\right|^{2} \\
& \quad-3 v_{k n} \sum_{p \neq k}\left|v_{p n}\right|^{2}-3 v_{k n} \sum_{s \neq n}\left|v_{k s}\right|^{2}, \quad k, n=1,2, \ldots, \\
2 \frac{d v_{k 0}}{d \tau}= & v_{k 0}-\frac{3}{2} v_{k 0}\left|v_{k 0}\right|^{2}-\bar{v}_{k 0} v_{0 k}^{2} \\
& -2 v_{k 0} \sum_{p \neq k}\left|v_{p s}\right|^{2}-3 v_{k 0} \sum_{s \neq 0}\left|v_{k s}\right|^{2}, \quad k=1,2, \ldots,
\end{aligned}
$$




$$
\begin{aligned}
2 \frac{d v_{0 n}}{d \tau}= & v_{0 n}-\frac{3}{2} v_{0 n}\left|v_{0 n}\right|^{2}-\bar{v}_{0 n} v_{n 0}^{2} \\
& \quad-2 v_{0 n} \sum_{s \neq n}\left|v_{p s}\right|^{2}-3 v_{0 n} \sum_{s \neq 0}\left|v_{s n}\right|^{2}, \quad n=1,2, \ldots, \\
2 \frac{d v_{00}}{d \tau}= & v_{00}-v_{00}\left|v_{00}\right|^{2}-2 v_{00} \sum_{s=p \neq 0}\left|v_{p s}\right|^{2} .
\end{aligned}
$$

Система (6)-(9) дополняется аналогичными уравнениями для $\bar{v}_{k n}, k, n=0,1, \ldots$

3. Краевая задача (1) при любом выборе области имеет пространственно однородный цикл, устойчивость которого просто обосновьвается без привлечения метода квазинормальных форм.

ТЕорема. Среди автомодельных ииклов и торов системы (6)-(9), у которых ненулевыми являются только координаты с не равными одновременно нулю индексами $k, n \leqslant \mathscr{N}_{0}$, орбитально әкспоненчиально устойчивы пространственно неоднородные ииклы, связанные с $v_{k n} u v_{n k}, k \neq n$.

ДокАЗАТЕЛЬСТво теоремы достаточно громоздко. Поэтому ограничимся важным случаем, когда некоторые $v_{k 0}$ и $v_{0 k}$ отличны от нуля, а остальные переменные системы (6)-(9) являются нулевыми. При этом условии после соответствующего сужения уравнений $(7),(8)$ приходим к системе

$$
\begin{aligned}
2 \dot{r}_{k 0} & =\left(1-\frac{3}{2} r_{k 0}^{2}-2 r_{0 k}^{2}-r_{0 k}^{2} \cos 2 \alpha_{k 0}\right) r_{k 0} \\
2 \dot{r}_{0 k} & =\left(1-\frac{3}{2} r_{0 k}^{2}-2 r_{k 0}^{2}-r_{k 0}^{2} \cos 2 \alpha_{k 0}\right) r_{0 k} \\
2 \dot{\alpha}_{k 0} & =\left(r_{k 0}^{2}+r_{0 k}^{2}\right) \sin 2 \alpha_{k 0},
\end{aligned}
$$

где $v_{k 0}=r_{k 0} \exp \left(i \varphi_{k 0}\right), v_{0 k}=r_{0 k} \exp \left(i \varphi_{0 k}\right), \alpha_{k 0}=\varphi_{k 0}-\varphi_{0 k}$. Из (12) следует, что $2 \alpha_{k 0} \rightarrow \pi$ при $\tau \rightarrow \infty$. Это обстоятельство позволяет перейти от $(10),(11)$ к системе

$$
\dot{\rho}_{k 0}=\left(1-\frac{3}{2} \rho_{k 0}-\rho_{0 k}\right) \rho_{k 0}, \quad \dot{\rho}_{0 k}=\left(1-\frac{3}{2} \rho_{0 k}-\rho_{k 0}\right) \rho_{0 k},
$$

где $\rho_{k 0}=r_{k 0}^{2}, \rho_{0 k}=r_{0 k}^{2}$, аттрактор которой - состояние равновесия $(2 / 5,2 / 5)$.

Итак, при анализе системы (6)-(9) переменные можно считать вещественными, если изменить знак перед третьими слагаемьми в уравнениях (6)-(8). При этом удобно перейти к переменным $\rho_{k n}=v_{k n}^{2}, k, n=0,1, \ldots$, что сводит проблему к анализу устойчивости ненулевых состояний равновесия получающейся системы. Эти замечания превращают доказательство теоремы в техническое, хотя и трудоемкое упражнение.

Отметим, что аналогичное утверждение справедливо и для второго случая, если $l_{1}-l_{2}=\gamma \varepsilon$, а постоянная $\gamma$ достаточно мала.

4. Представляет интерес рассмотрение данного вопроса для сингулярно возмущенной краевой задачи

$$
u_{t t}-2 \varepsilon u_{t}+u=2 \varepsilon \mu \Delta u+f\left(u, u_{t}\right),\left.\quad \frac{\partial u}{\partial \nu}\right|_{\Gamma}=0
$$


где параметр $\mu>0$, а гладкая функция $f$ имеет в нуле высший порядок малости. Обозначим через $d$ первую ляпуновскую величину обыкновенного уравнения $\ddot{u}+u=f(u, \dot{u})$. Считаем, что $\operatorname{Re} d<0$. Если $\operatorname{Im} d>0$, однородньй цикл краевой задачи (13) орбитально экспоненциально устойчив при любом изменении $\mu$.

Пусть $d=-1-i \omega^{2}$. Тогда квазинормальная форма краевой задачи (13) имеет вид

$$
\frac{\partial \xi}{\partial \tau}=-i \mu \Delta \xi+\xi-\left(1+i \omega^{2}\right)|\xi|^{2} \xi,\left.\quad \frac{\partial \xi}{\partial \nu}\right|_{\Gamma}=0
$$

Краевая задача для $\bar{\xi}$ получается из (14) путем применения операции комплексного сопряжения. Если в (14) оператор Лапласа заменить его какой-то разностной аппроксимацией, то при малых $\mu$ единственным аттрактором получающейся системы обыкновенных уравнений является многомерньй тор. Поэтому квазинормальную форму теперь уже краевой задачи (14) следует строить следующим образом.

Положим в (14)

$$
\begin{gathered}
\xi=\left(1+\mu \Phi_{1}(\phi)+\mu^{2} \Phi_{2}(\phi)\right) \exp (i \phi) \\
\frac{\partial \phi}{\partial \tau}=-\omega^{2}+\mu \Pi_{1}(\phi)+\mu^{2} \Pi_{2}(\phi),\left.\quad \frac{\partial \phi}{\partial \nu}\right|_{\Gamma}=\left.\frac{\partial \Delta \phi}{\partial \nu}\right|_{\Gamma}=0
\end{gathered}
$$

Подставляя (15), (16) в (14), заключаем, что

$$
\begin{aligned}
\Phi_{1} & =\frac{1}{2} \Delta \phi, & \Pi_{1} & =-\omega^{2} \Delta \phi+\phi_{x}^{2}+\phi_{y}^{2}, \\
\Phi_{2} & =\frac{\omega^{2}}{4} \Delta^{2} \phi-\frac{5}{8}(\Delta \phi)^{2}, & \Pi_{2} & =-\frac{\omega^{4}+1}{2} \Delta^{2} \phi+\omega^{2}(\Delta \phi)^{2} .
\end{aligned}
$$

Из (17), (18) следует, что заведомо сложно устроены аттракторы краевой задачи (16).

\section{СПИСОК ЦИТИРОВАННОЙ ЛИТЕРАТУРЫ}

[1] Колесов Ю.С. Проблема аттракторов нелинейных волновых уравнений в плоских областях // Матем. заметки. 2000. Т. 68. № 2. С. 217-229. 